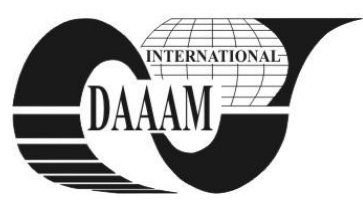

Annals of DAAAM for 2012 \& Proceedings of the 23rd International DAAAM Symposium, Volume 23, No.1, ISSN 2304-1382 ISBN 978-3-901509-91-9, CDROM version, Ed. B. Katalinic, Published by DAAAM International, Vienna, Austria, EU, 2012 Make Harmony between Technology and Nature, and Your Mind will Fly Free as a Bird Annals \& Proceedings of DAAAM International 2012

\title{
ON EVALUATION APPROACH OF STRUCTURAL INTEGRITY LEVEL FOR BRIDGE BEARINGS IN RESPECT WITH IMPACT DYNAMIC TEST RESULTS
}

\author{
LEOPA, A[drian]; DEBELEAC, C[armen] N[icoleta] \& NASTAC, S[ilviu]
}

\begin{abstract}
This study deals with area of structural health monitoring of bridges and viaducts, and especially of insulation systems for these kinds of constructions. Analysis in this paper reveals some particular aspects regarding the structure response of the impact tests applied on deck bridges in view of bearings damage level characterization. Basic information in this paper derives from practical cases of viaducts on A3 Highway in Romania. Final results will be included into a regulated procedural methodology for continuous monitoring of bridges damage level.
\end{abstract}

Keywords: bridge health monitoring, structural integrity, rubber-based bearings, vibration isolation.

\section{INTRODUCTION}

Monitoring of bridges health level and evaluation of their structural integrity necessitate an appropriate method which has to be able to assure a set of essential requirements as follows: evaluation of main parameters regarding the bridge dynamics and significant changes of these during the exploitation time, prominence of direct influence of ageing about the damage imminence both into the deck bridge structure, and into the bearings, prominence of external perturbation factors influences about the bridge structure and the dynamic isolation systems, evaluation of global damage level in respect with its reference limit and mean time to fail values. Final purpose of these evaluations consists to an efficient maintenance procedure especially for dynamic isolation devices.

The analysis proposed in this paper start from the basic method of the impact test of deck bridges with truck tip movement over a regular bump. The instrumental tests of dynamic behaviour dignify the spectral characteristic of the structure and reveal the possible resonance area. Taking into account the rubberbased bearings it offers the premises to evaluate the isolation performances against vibration and shocks due to road traffic and also seismic actions [1...7].

Utilization of truck tips for dynamic excitation is able to induce different forces into the bridge structure as a function of truck masses geometry and loads. This type of external excitation can be assimilates such a random or such a deterministic perturbation, depending the way to generate the signal. Also, the spectral composition of excitation signal can lead to a weighted response - when the excitation can be assimilates with an impulsive load, or to a harmonic response - when the excitation is suppose to be a simplified (much theoretical) harmonic signal [5...7].

The theoretical approaches in this paper presents uncommon situations which can appears in case of impact test serviceable procedure. It has to be mentioned that these phenomenon can be either efficacious or inefficacious depending the type of excitation and of the response wanted to use into the experimental tests.

\section{BASIC MODEL}

This paragraph presents the basic model of the proposed method for dynamic evaluation of the bridge structural response. The scheme in Fig. 1 depicts the physical model of a bridge section with a truck tip moving over and with a regular bump mounted at a certain distance from one of the section end.

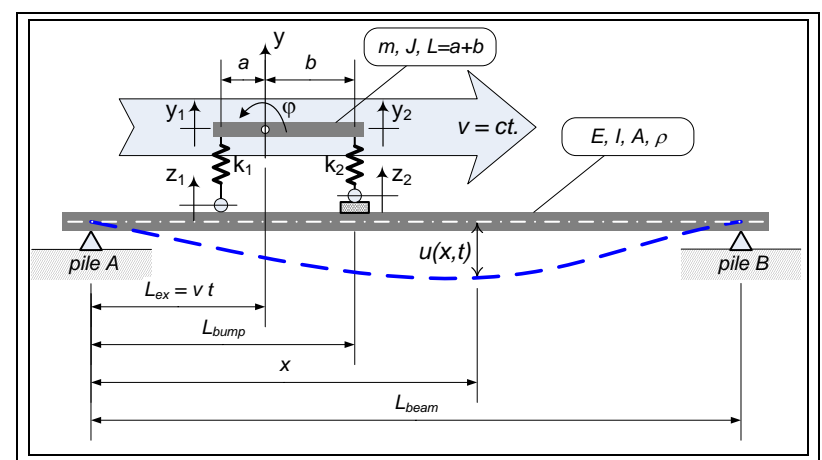

Fig. 1. Schematic diagram of deck bridge dynamic testing

Resulting from the model in Fig. 1 the schematization supposes an elastic beam insulated at both ends with a regular bump mounted at $L_{\text {bump }}$ from one of the end. The truck model supposes a rigid mass with two degrees of freedom linked with $k_{i}$ rigidities by the beam. The position of this mass $L_{e x}$ relative to the beam end is variable in respect with the constant velocity $v$ of the truck. In Fig. 1 schematic diagram $u(x, t)$ denotes the beam deflection, $z_{i}$ denote the vertical displacements of the wheels on the road surface, $y_{i}$ are the displacements of the two ends of the truck model, $y$ and $\varphi$ denote the independent coordinates of truck mass respectively in its center of gravity situated at $a$ and $b$ distances from each end.

The differential equations systems of the model can be assembled based on the equation of transversal wave in beams and using Laplace 2nd order equation for the truck mass. Hereby the movement of the bridge section can be evaluate with the partial differential equation

$$
\left[E(x) I(x) u^{\prime \prime}(x, t)\right]^{\prime \prime}+m_{v}(x) A(x) \ddot{u}(x, t)=q(x, t)
$$

where $E, I, m_{v}, A$ denote Young's modulus, moment of inertia, volumetric specific mass, respectively sectional 
area of the deck bridge as variable parameters in respect with longitudinal position $x$, and $q(x, t)$ denotes the external excitation term.

The movement of the truck tip over the bridge section can be simulated with the next differential equations system

$$
\left\{\begin{array}{l}
m \ddot{\delta}_{i} \frac{L-\eta_{i}}{L}+k_{i} \delta_{i}=-m\left(\ddot{z}_{i} \frac{L-\eta_{i}}{L}+\ddot{u}\left(x_{i}, t\right)\right) \\
\frac{J}{L}(-1)^{i} \ddot{\delta}_{i}+(-1)^{i} k_{i} \eta_{i} \delta_{i}=-\frac{J}{L}(-1)^{i}\left(\ddot{z}_{i}+\ddot{u}\left(x_{i}, t\right)\right)
\end{array}\right.
$$

where $m, J, L$ denote the mass, moment of inertia, respectively total length of the truck tip, $\eta_{i}$ is the distance between the center of gravity and each end of truck, and $\delta_{i}$ denote the deformations of each wheel. The equations of system (2) suppose summation for $i=1,2$ according the two axles of the truck. Simplified notations in (2) have the significance as follows

$$
\left\{\begin{array}{l}
a=\eta_{1} \\
b=\eta_{2}=L-\eta_{1} \\
\delta_{i}=y_{i}-z_{i}-\left.u\left(x_{i}, t\right)\right|_{i=1,2}
\end{array}\right.
$$

The external perturbation function $q(x, t)$ contains both static and dynamic terms, and assure the linkage between the beam evolution and the truck dynamics. The expression of $q(x, t)$ can be written as follows

$$
\begin{aligned}
q(x, t) & =q_{B}(x, t)+q_{e x_{-} s t}(x, t)+q_{e x_{-} d y n}(x, t)= \\
& =m_{v}(x) A(x) g+\cdots \\
& \cdots+\left\{\begin{array}{l}
\frac{m g}{L}\left(L-\eta_{i}\right)+\frac{k_{i} \delta_{i}}{L}, x=L_{e x} \mp \eta_{i} \\
0, \text { otherwise }
\end{array}\right.
\end{aligned}
$$

The terms in (4) correspond by beam weight, static and dynamic external loads, and also respect the summation for $i=1,2$ according the two axles of the truck.

The hypothesis of constant characteristics of material and masses geometry for bridge section leads to a simplified form of dynamic equations as follows

$$
\left\{\begin{array}{l}
E I\left(u^{\prime \prime}\right)^{\prime \prime}+m_{v} A \ddot{u}=m_{v} A g+\cdots \\
\cdots+\left\{\begin{array}{l}
\frac{m g}{L}\left(L-\eta_{i}\right)+\frac{k_{i} \delta_{i}}{L}, x=L_{e x} \mp \eta_{i} \\
0, \text { otherwise }
\end{array}\right. \\
m \ddot{\delta}_{i} \frac{L-\eta_{i}}{L}+k_{i} \delta_{i}=-m\left(\ddot{z}_{i} \frac{L-\eta_{i}}{L}+\ddot{u}_{i}\right) \\
\frac{J}{L}(-1)^{i} \ddot{\delta}_{i}+(-1)^{i} k_{i} \eta_{i} \delta_{i}=-\frac{J}{L}(-1)^{i}\left(\ddot{z}_{i}+\ddot{u}_{i}\right)
\end{array}\right.
$$

with simplified notations

$$
\| \begin{aligned}
& a=\eta_{1} \\
& b=\eta_{2}=L-\eta_{1} \\
& \delta_{i}=y_{i}-z_{i}-\left.u\left(x_{i}, t\right)\right|_{i=1,2} \\
& \ddot{u}_{i}=\ddot{u}\left(x_{i}, t\right)
\end{aligned}
$$

\section{PERTURBATION SIGNAL ANALYSIS}

Taking into account the sub-model of the excitation system (truck tip equipment) results that analysis of perturbation signal structure can be developed with the help of two degrees of freedom dynamic model (see Fig.2).

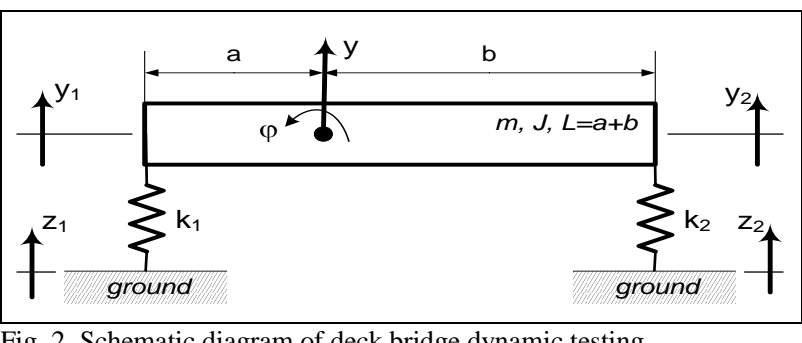

Fig. 2. Schematic diagram of deck bridge dynamic testing

The natural pulsations of the simplified model like that depicted in Fig. 2 can be evaluated with the homogeneous differential equations system, as follows

$$
\begin{aligned}
& m\left[\ddot{\delta}_{1}(1-\xi)+\ddot{\delta}_{2} \xi\right]+k_{1} \delta_{1}+k_{2} \delta_{2}=0 \\
& \frac{J}{L}\left(-\ddot{\delta}_{1}+\ddot{\delta}_{2}\right)-k_{1} L \xi \delta_{1}+k_{2} L(1-\xi) \delta_{2}=0
\end{aligned}
$$

where the center of gravity coordinates was written in respect with variable changes as follows

$$
\left\{\begin{array}{l}
a=L \xi \\
b=L(1-\xi)
\end{array}\right.
$$

Supposing the solutions of (7) as follows

$$
\| \begin{aligned}
& \delta_{1}=a_{1} \sin \left(\omega_{n} t+\theta\right) \\
& \delta_{2}=a_{2} \sin \left(\omega_{n} t+\theta\right)
\end{aligned}
$$

making derivatives of (9), replacing into (7) and grouping the terms results an algebraic equations system with two unknown variables as $a_{1}, a_{2}$

$$
\left\{\begin{array}{l}
a_{1}\left[k_{1}-m \omega_{n}^{2}(1-\xi)\right]+a_{2}\left(k_{2}-m \omega_{n}^{2} \xi\right)=0 \\
-a_{1}\left(k_{1} L \xi-\frac{J}{L} \omega_{n}^{2}\right)+a_{2}\left[k_{2} L(1-\xi)-\frac{J}{L} \omega_{n}^{2}\right]=0
\end{array}\right.
$$

which have the non-zero solutions in case of null determinant $\left(\Delta_{*}=0\right)$

$$
\Delta_{*}=\left|\begin{array}{cc}
k_{1}-m \omega_{n}^{2}(1-\xi) & k_{2}-m \omega_{n}^{2} \xi \\
-k_{1} L \xi+\frac{J}{L} \omega_{n}^{2} & k_{2} L(1-\xi)-\frac{J}{L} \omega_{n}^{2}
\end{array}\right|
$$


With the ratio between rigidities noted by $\alpha=k_{2} / k_{1}$ into (11) results the characteristic equation of natural pulsations in respect with $\left(m, J, L, \eta, \alpha, k_{l}\right)$ parameters

$$
\omega_{n}^{4}-\frac{k_{1} L^{2}}{J}\left[(\xi-1)^{2} \alpha+\frac{J}{m L^{2}}(\alpha+1)+\xi^{2}\right] \omega_{n}^{2}+\frac{k_{1}^{2} L^{2} \alpha}{m J}=0
$$

Taking into account the last equation and following the initial purpose of this paragraph results that the inequality between the squared values of natural pulsations can be evaluated as follows

$$
\begin{aligned}
\varepsilon & =\omega_{n 1}^{2}-\omega_{n 2}^{2}= \\
& =\sqrt{\left(\frac{k_{1} L^{2}}{J}\left[(\xi-1)^{2} \alpha+\frac{J}{m L^{2}}(\alpha+1)+\xi^{2}\right]\right)^{2}-4 \frac{k_{1}^{2} L^{2} \alpha}{m J}}
\end{aligned}
$$

Optimization of the mass geometry configuration with respect in position of the center of gravity $\xi$ and stiffness ratio $\alpha$ imposes an appropriate estimation of basic parameters values. Without any significant diminishing of general character of this analysis it will be consider that the truck tip equipment have a mass range between $(30000 \ldots 50000) \mathrm{kg}$ and a wheels rigidity having $k_{l}=100000 \mathrm{~N} / \mathrm{m}$.
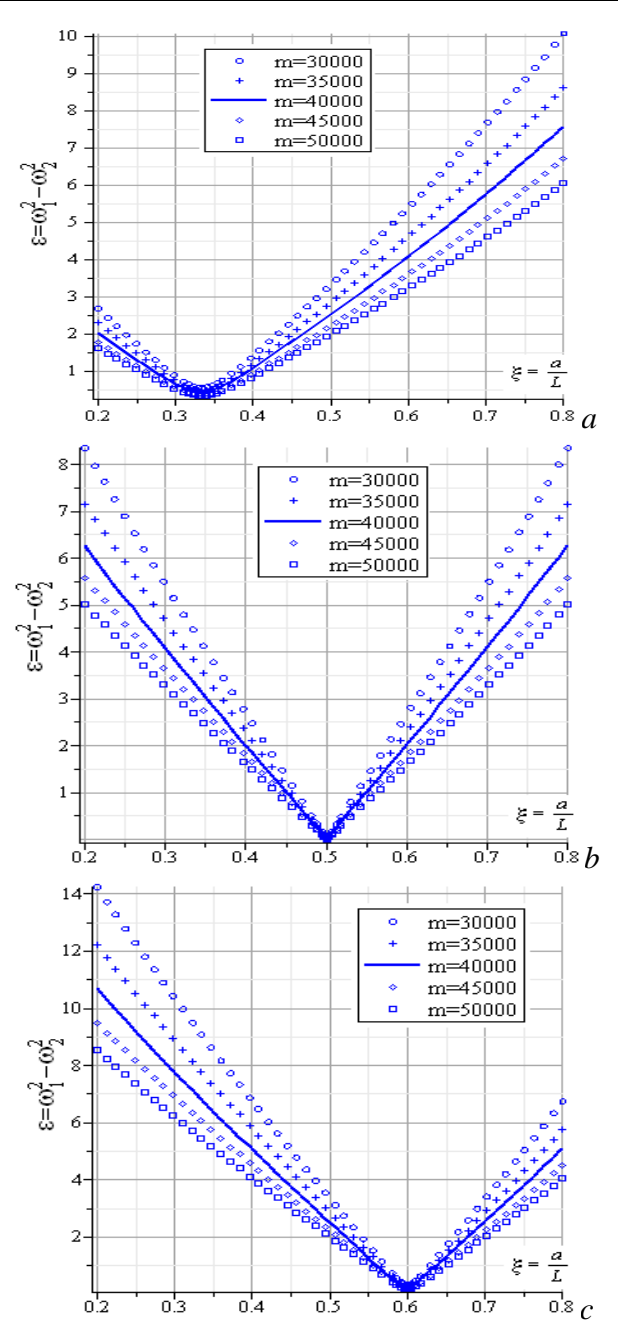

Fig. 3. Evolution of natural pulsation difference in respect with $\xi$ and $m$ parameters for (a) $\alpha=0.5$, (b) $\alpha=1.0$ and (c) $\alpha=1.5$
Simulations presented in Figs. 3...5 shows the evolution of the natural pulsation difference $\varepsilon$ as a function of the relative position $\xi$, the rigidity ratio $\alpha$ and five main values of mass $m$.

Diagrams in Fig. 3 show the evolution of the $\varepsilon$ parameter in respect with the relative position $\xi$, according with discrete variation of mass $m$ and rigidity ratio $\alpha$. These diagrams dignify the system behaviour into the area of small differences of $\varepsilon$ and also reveal possible null values of natural pulsation difference.

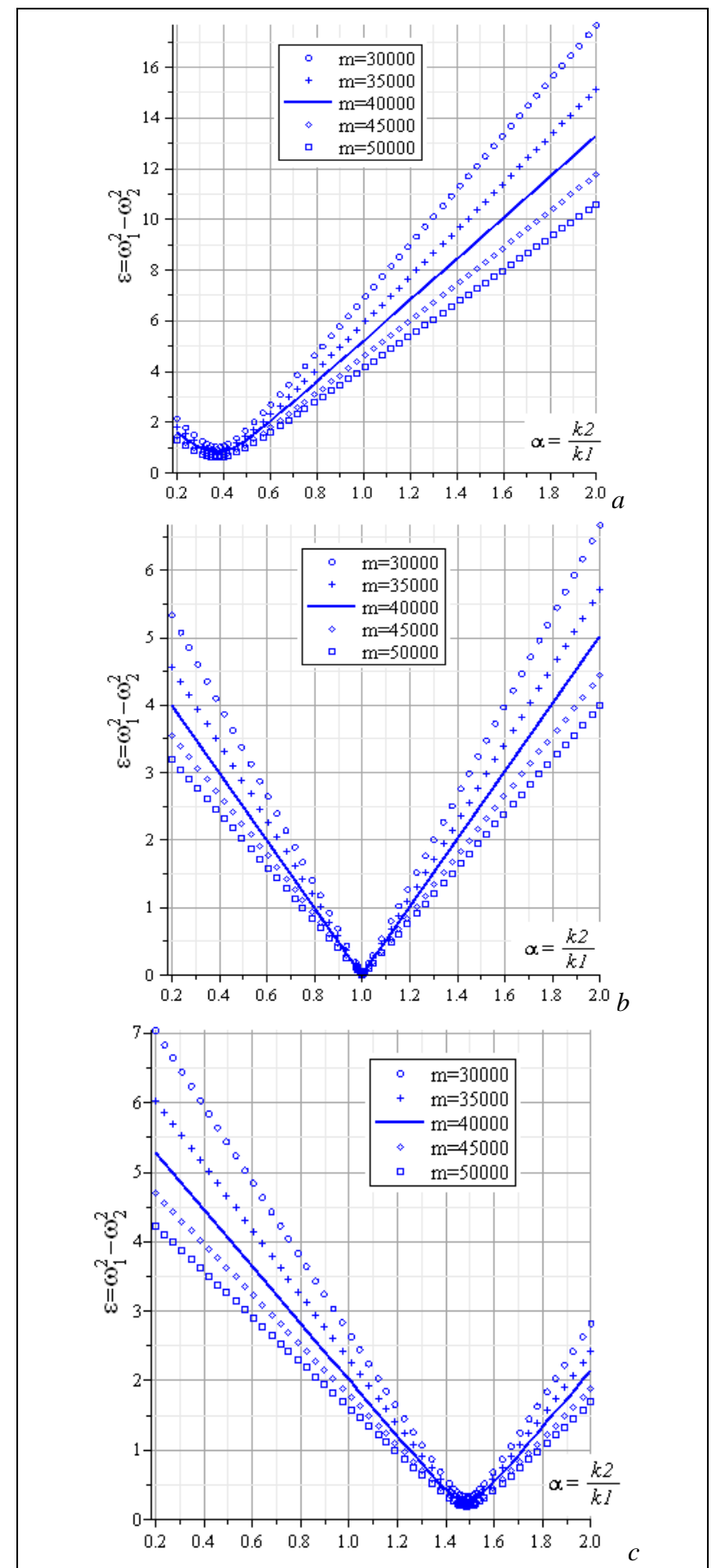

Fig. 4. Evolution of natural pulsation difference in respect with $\alpha$ and $m$ parameters for (a) $\xi=0.25$, (b) $\xi=0.5$ and (c) $\xi=0.6$ 


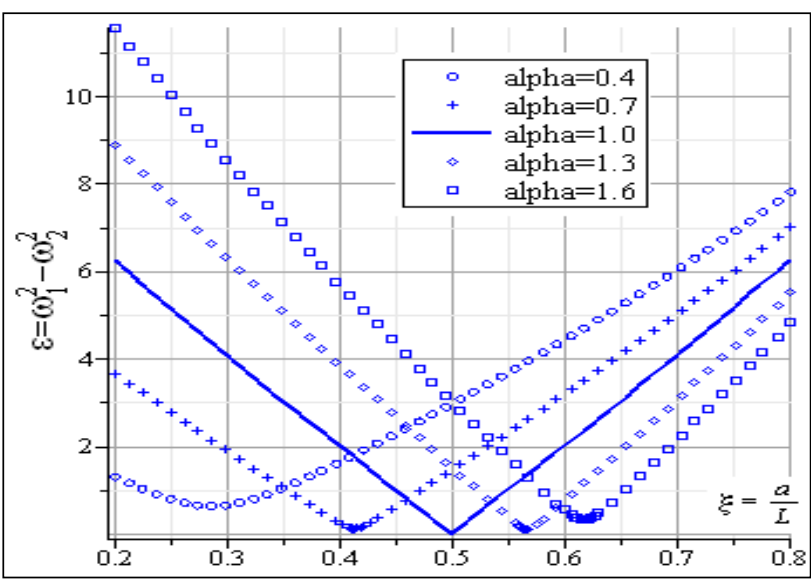

Fig. 5. Evolution of natural pulsation difference in respect with $(\xi, \alpha)$ for $m=40000 \mathrm{~kg}$

An appropriate analysis with previous case results from the diagrams in Fig. 4. These graphs correspond with the evolution of the natural pulsation difference in respect with the rigidity ratio $\alpha$, according with discrete variation of mass $m$ and relative position $\xi$.

According the previous analysis the diagram in Fig.5 presents the evolution of the natural pulsation difference $\varepsilon$ parameter as a function of the relative position $\xi$ and the rigidity ratio $\alpha$ for mass $m$ of $40000 \mathrm{~kg}$. This value was adopted according with the total mass of the truck tip used into the instrumental tests. It had to be mentioned that the entire simulation procedure supposing the rigidity ratio $\alpha$ into a range of $(0.2 \ldots 2.0)$ and the relative position $\xi$ having limit values of 0.2 , respectively 0.8 . Greater values of rigidity ratio have not a serviceable purpose.

\section{DISCUSSIONS AND PARTIAL REMARKS}

The analysis of the natural frequencies dispersion and distribution of the excitation system is very useful to evaluate the characteristic of the perturbation type into the bridge and also the dynamic behaviour of the deck bridge and its bearings right away the impulsive load have been produced. The truck tip equipment had to working in post-resonance domain according with its main purpose into this analysis that is the excitation system. Also the impulsive test of the deck bridges have not to affects this kind of excitation system.

One of the basic ideas of this study supposes to restrain the spectral spilling of the natural frequencies of excitation. Hereby it will reduce the width of possible resonance area. The entire study follows the hypothesis of best minimizing of the natural pulsations difference parameter $\varepsilon$ thus that for a certain reduction of the reference pulsation it will be simply left-side shifted the entire resonance area. In presented case the rigidity $k_{l}$ is the appropriate element to do this intention through different technical means.

According these hypotheses lets briefly evaluate the presented results. From the diagrams depicted in Figs. 3 to 5 results that unitary ratio of stiffness and symmetrical center of gravity provides a superposition of both natural pulsations. It also results than greater masses imply reduced values of $\varepsilon$ on the entire simulated domain. The direct linkage between stiffness ratio and relative position of the center of gravity (CG) of truck tip equipment is mainly sustained by the graphs in Fig. 5. It can be observed that in the same time with stiffness ratio $\alpha$ reduction, results also a reduction of the CG relative position $\xi$ for the minimum value of $\varepsilon$ parameter. But the absolute minimum value of $\varepsilon$ increases in respect with dispersion of $\alpha$ parameter from unitary value, regardless the dispersion direction. Analysis of the $(\alpha, \xi)$ dependences depicted in Figs. 3 and 4 intensify the previous observations.

\section{CONCLUSION}

Major purpose of this study consisted by presentation and validation of some practical possibilities to adjust the characteristic of the excitation system usually used in bridge bearings health monitoring procedures without reducing its main capabilities but helping it to work into the appropriate domain regarding initial requirements. Taking into account the remarks in previous paragraphs it had to be concluded that optimization of main configuration of the masses geometry provides a serviceable way to control dynamic effects induced into a deck bridge and its bearings during the experimental tests and analysis of the damage level.

\section{ACKNOWLEDGEMENTS}

This work was supported by UEFISCDI (CNCSISUEFISCSU), project number PN II-RU-PD code $597 / 2010$

\section{REFERENCES}

[1] Bratu P. (2008). Theoretical Mechanics, Impuls House Publishing, Romania

[2] Debeleac C. (2006). Dynamic Behaviour Analysis of Speedy Frontal Loader on View of Qualities Performances Established, Ph.D. Dissertation, Dunarea de Jos University of Galati, Romania

[3] Leopa, A., (2011). The Impulsive Loading Influence on Dynamic Response Parameters of the Viaduct Type Structure, The Annals of "Dunarea de Jos" University of Galati, Fascicle XIV Mechanichal Engineering, No2, 2011, ISSN 1224-5615, pp.65-67

[4] Leopa, A., Nastac, S., Dragan, N., Debeleac, C., (2011). Considerations on the Influence of Viscoelastic Behavior of Nonlinear Systems Bearing on the Dynamic Constructions Response, Proceedings of the International Conference on Structural Engineering Dynamics, Tavira, Portugalia, 2011, ISBN 978-989-96276-1-1

[5] Nastac S. (2004). Computational Engineering with Applications, Impuls House Publishing, Bucharest, Romania

[6] Nastac, S. (2008). Analysis of Working Performances at Damaged Vibration Isolation Devices (2008). 0949-0950, Annals of DAAAM for 2008 \& Proceedings of the 19th International DAAAM Symposium, pp 475, Editor B. Katalinic, Published by DAAAM International, Vienna, Austria 2008

[7] Nastac, Silviu (2008). Performances Evaluation at Damaged Vibration Isolation Devices, Chapter 47 in DAAAM International Scientific Book 2008, pp.551-564, B. Katalinic (Ed.), Published by DAAAM International, Vienna, Austria 